\title{
Preliminary Design and Optimization of Toroidally-Wound Limited Angle Servo Motor Based on a Generalized Magnetic Circuit Model
}

\author{
Shuai $\mathrm{Wu}^{123}$, Xiangyu Zhao ${ }^{1}$, Xiao $\mathrm{Li}^{1}$, Patrick C. K. Luk ${ }^{3}$, and Zongxia Jiao ${ }^{12}$ \\ ${ }^{1}$ School of Automation Science and Electrical Engineering, Beihang University, Beijing, 100191, China \\ ${ }^{2}$ Science and Technology on Aircraft Control Laboratory, Beihang University, Beijing, 100191, China \\ ${ }^{3}$ Electric Power and Drives Group, Power Engineering Centre, Cranfield University, Cranfield, MK43 0AL, U.K.
}

\begin{abstract}
This paper proposes a new generalized equivalent magnetic circuit model for the preliminary design of a toroidally-wound limited angle servo motor (LASM). In the model, the magnetic networks are formulated as a function of the pole number and geometric dimensions. Nonlinear saturation effect of the ferromagnetic material is also taken into consideration. A multi-objective optimization function involving the torque requirement, the mass, the time constant, and magnetic saturations of ferromagnetic material is introduced. Based on the proposed model, six design cases with different objectives have been carried by the particle swarm optimization (PSO) method. The comparisons of different optimization cases demonstrate the effectiveness and computation efficiency of the proposed method, and hence its suitability in preliminary design. Moreover, the generalized model can be readily applied in the other electromagnetic modeling.
\end{abstract}

Index Terms-Magnetic equivalent circuit, limited angle servo motor, magnetic saturation, multi-objective optimization.

\section{INTRODUCTION}

The limited angle servo motor (LASM) is an electromechanical actuator which can rotate in a limited angle normally less than $180^{\circ}$. The LASM is widely used in scanning systems, hard disks, servo valve and other servo systems that require limited rotational motion. It usually requires high control precision and high dynamic response. Therefore, conventional alternating current (AC) or brushless permanent magnet (BLPM) motors are not suitable due to the inherent cogging torque and low positional precision. Various types of direct current (DC) based electromagnetic devices for limited motion are well reported in the literature [1]. It is noted that the torque per unit current of the toroidal winding on the stator and permanent-magnet rotor type as shown in Fig. 1, is largely invariant with respect to the rotor position over the working angular range.

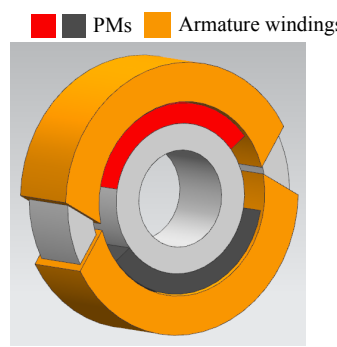

(a) Two-Pole

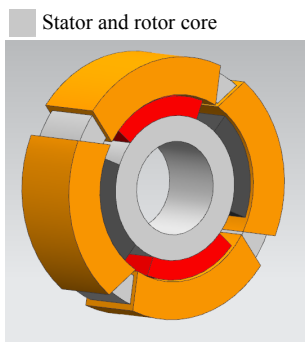

(b) Four-Pole
Fig. 1. Structure of toroidal type with a distributed winding on the stator and a permant-magnet rotor. (a) two pole design, (b) four pole design.

The preliminary design of the LASM should give a general layout of the key machine parameters including pole number, winding conductor diameter, and geometrical dimensions, based on the application with specific parameters such as angular range, torque constant, and maximum winding resistance.

Manuscript received XXX XX, 2015; revised XXX XX, XXXX. Corresponding author: Shuai Wu (email: ws@buaa.edu.cn).
The equivalent magnetic circuit model (EMCM) is a widely used technique and often serves as the first step in the analysis and design of electric machines [2-5]. In magnetic circuits, magnetic reluctance is analogous to resistance, and flux is analogous to current. The excitation of magnetic circuits is ampere-turns, analogous to voltage. Therefore, the magnetic field characteristics can be obtained using electric circuit principle, such as Kirchhoff's voltage law (KVL) and Kirchhoff's current law (KCL). Due to over simplifications, conventional EMCM generally cannot provide accurate results. Therefore, EMCM is usually only employed for preliminary design, and often incorporated with other methods such as finite-element analysis (FEA) for further confirmation of results $[6,7]$.

FEA can provide accurate magnetic field predication, but the entire process is often computationally intensive and therefore costly. However, FEA optimization process can also be rather limited as only geometrical dimensions can be changed automatically, whilst any modification on machine parameters (such as pole numbers) during preliminary design would require the model to be reconstructed manually. As the number of parameters needed to be decided in the preliminary phase can be very large, this will result in generation of huge amount of possible solutions. For these reasons, FEA is not considered suitable in preliminary design. Thus, the use of EMCM in preliminary design study has been well documented, and a detailed development history of EMCM is given in [7].

An optimum procedure is required for preliminary design to reduce the weight, improve the dynamic performance, and other performance index, in addition to meeting the application requirements. It is therefore a multi-objective optimization design problem. In $[8,9]$, a simple EMCM model of a LASM is developed and hierarchy process (AHP) or axiomatic design (AD) methods are used to simplify the design procedure. But only a two pole design is studied, and the AHP or AD algorithm are known to be suitable for comparing existing solutions rather than for preliminary design. Evolutionary 
type artificial intelligence methods which can optimize design parameters automatically have been used in many studies. A differential evolutionary (DE) multi-objective optimization of a surface mounted PM actuator is proposed in [10]. The $\mathrm{DE}$, genetic algorithm (GA) and simulated annealing (SA) methods are used in synchronous reluctance motors multiobjective optimization [11]. An orthogonal multi-objective chemical reaction optimization approach for the brushless DC motor design is proposed in [12].

The main aim of this paper is to propose a new generalized, time-efficient and yet accurate EMCM model of a LASM for preliminary design and optimization. The proposed generalized model is a major contribution to existing design tools. The paper is organized as follows. Section II discusses the analytical modeling in which the pole number and all critical geometrical dimensions are set as variable and therefore can be optimized automatically. The flux saturation is taken into account according to the B-H curve of the material so that the flux can be accurately obtained. The generalized model is arranged in KCL matrix format so that it is easy to be extended and solved. In section III, the accuracy of the model is evaluated by FEA method. Section IV discusses and classifies the design objectives of the LASM. A generalized multiobjective function is then constructed for optimization. The particle swarm optimization (PSO) method is applied in the present study in six different design cases for the verification of the proposed method. Section V provides the concluding remarks.

\section{Analytical Modeling}

\section{A. Geometry definitions and magnetic circuits}

In the EMCM, the accuracy of the model requires careful study of the magnetic circuits. The geometry definitions of a four pole LASM are shown in Fig. 2. The general torque characteristic for the LASM is presented in Fig. 3. The constant torque region is $\pm \alpha_{1}$ which depends on the arc angle of each sector of armature winding and the pole PM arc angle:

$$
\alpha_{1}=\frac{\phi_{c}-\phi_{m}}{2}
$$

The equivalent magnetic circuits of the LASM is shown in Fig. 4. The symbols in Fig. 4 include absolute magnetomotive force $F$, magnetic reluctance $R$, magnetic flux $\phi$. The definitions of the subscript and superscript of these symbols are list as below.

$F_{r}^{0,1, \cdots, N-1}$ The MMF in the rotor of each divided sectors.

$F_{m}^{0,1, \cdots, N-1}$ The MMF on the junction surface of permanent magnet and air gap of each divided sectors.

$F_{s}^{0,1, \cdots, N-1}$ The MMF in the stator of each divided sectors.

$R_{r}^{0,1, \cdots, N-1}$ Reluctances between node $F_{r}^{n}$ to $F_{r}^{n+1}$.

$R_{m}^{0,1, \cdots, N-1}$ Reluctances between node $F_{r}^{n}$ to $F_{m}^{n}$.

$R_{g}^{0,1, \cdots, N-1}$ Reluctances between node $F_{m}^{n}$ to $F_{s}^{n}$.

$R_{s}^{0,1, \cdots, N-1}$ Reluctances between node $F_{s}^{n}$ to $F_{s}^{n+1}$.

$R_{m c}^{0,1, \cdots, N-1}$ Reluctances between node $F_{m}^{n}$ to $F_{m}^{n+1}$.

$\phi_{m}^{0,1, \cdots, N-1}$ Fluxes produce by the PM of each divided sectors.

$\phi_{c}^{0,1, \cdots, N-1}$ Fluxex produce by the windings of each divided sectors.

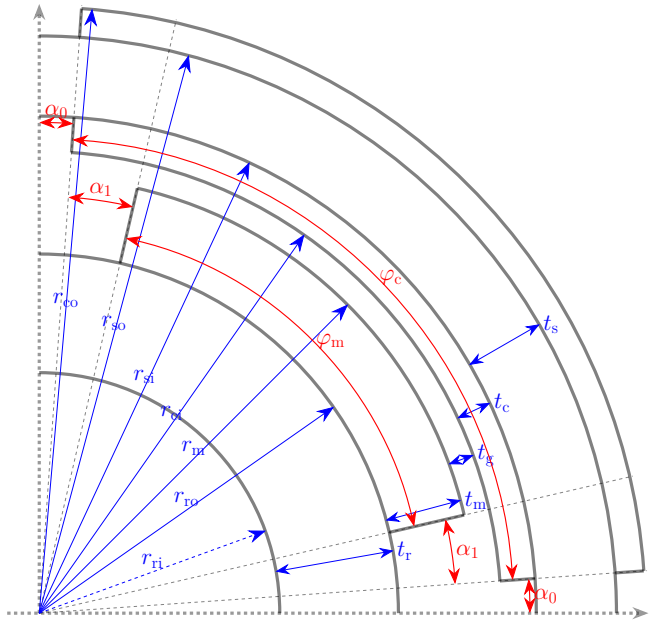

Fig. 2. Geometry definition of LASM. $\phi_{c}$ is the stator winding arc angle, $\phi_{m}$ is the pole PM arc angle, $\alpha_{0}$ is angle gap between two windings, $\alpha_{1}$ is the constant torque angle, $r_{r i}$ is radius of inner rotor, $t_{r}, t_{m}, t_{g}, t_{c}$ and $t_{s}$ are thickness of rotor, PMs, air-gap, winding and stator respectively.



Fig. 3. Torque versus rotor position characteristic of the LASM

The number $N$ is the total divided sections of the model. More divided sections result in higher accuracy but higher computational cost. It should be noted that, as the PM and coil are not continuously distributed in the circumferential direction, the divided sections are also not uniform. There are three different conditions which are presented in three different colors in Fig. 4. The red color areas are the sector of PM which is divided into 3 sections. The yellow areas that are the sector between PM and coil, which is the working range, are divided into 1 section. The blue areas are the empty sector between two neighbor poles which are divided into 1 section. And it should be noted that, in order to maintain consistency for the superscripts, all symbols are indexed from 1 to $N$. If a component does not exist in one section, then it is set to zero (for source) or infinite (for resistance). For example, there is no $\mathrm{PM}$ in the section 0 and 1 , then $\phi_{r m}^{0}, \phi_{r m}^{1}=0$.

Define the poles number is $N_{p}$, the count of divided sections of PM sector is $N_{m}$, the count of divided sections of working range is $N_{c}$, the count of divided sections of empty range between neighbor poles is $N_{e}$. These number can be set in calculation program to get the uniform model. In Fig. $4, N_{p}=$ 4, $N_{m}=3, N_{c}=1$, and $N_{e}=1$. Then the total sections number $N$ in Fig. 4 is $N_{p}\left(N_{m}+2 \times N_{c}+N_{e}\right)=2 \times(3+$ $2 \times 1+1)-1=20$. Other related parameters including the vacuum permeability $\mu_{0}$, effective axial length $L_{m}$.

Some assumptions are made to develop the mathematical model as:

- Permeability of the iron core is variable, which is ex- 


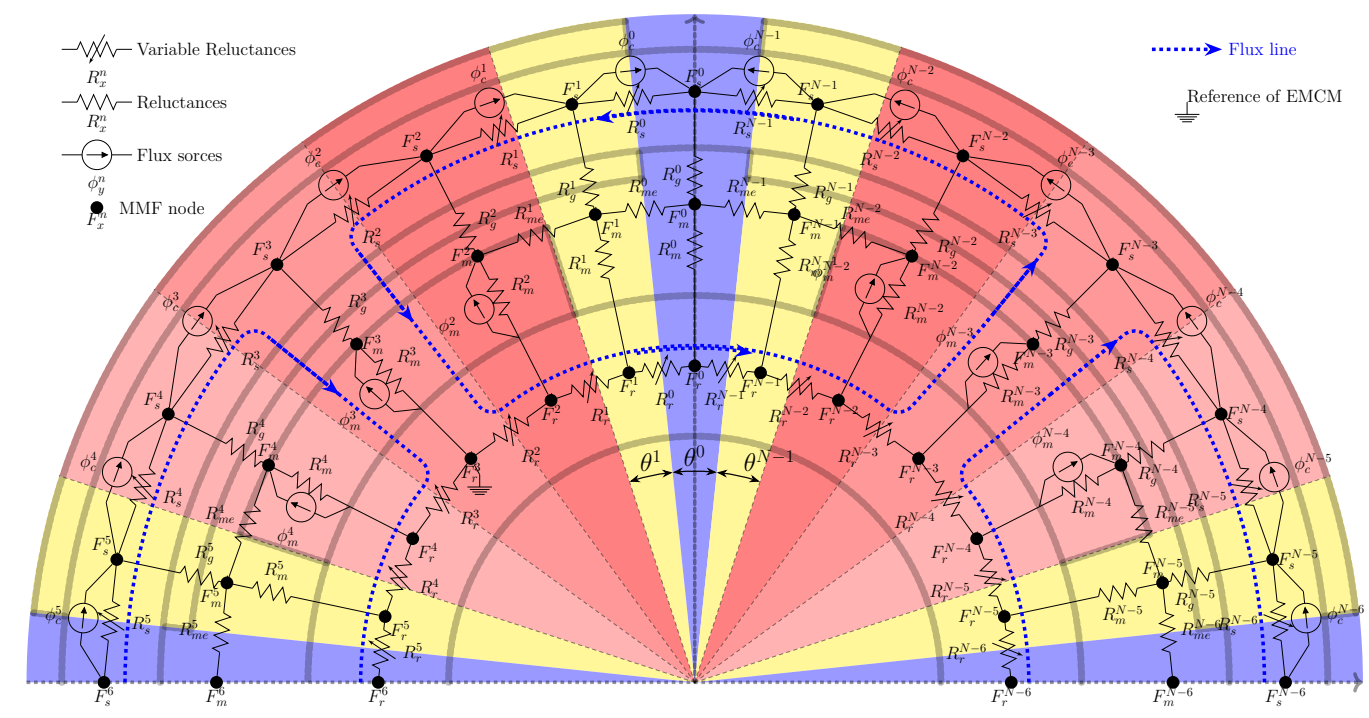

Fig. 4. Magnetic circuit mode of LASM. Only half of the full size mode are presented here since it is symmetric.

pressed as a variable resistance in Fig. 4.

- Flux flowing through each resistance is uniform.

- The leakage path of PM to the gap between two neighbor PMs is also considered.

As shown in Fig. 2 and Fig. 4, the reluctance in circumferential direction of $n$th stator segment $R_{s}^{n}$, $n$th air gap segment $R_{g}^{n}, n$th of segment of PM $R_{\mathrm{PM}}^{n}$, $n$th rotor segment $R_{\mathrm{r}}^{n}$ are expressed, respectively, as

$$
\begin{aligned}
& R_{s}^{n}=\frac{0.5\left(r_{\mathrm{so}}+r_{\mathrm{si}}\right) 0.5 \theta^{n}}{\mu_{\text {iron_s }}^{n} t_{\mathrm{s}} L_{\mathrm{m}}}+\frac{0.5\left(r_{\mathrm{so}}+r_{\mathrm{si}}\right) 0.5 \theta^{n+1}}{\mu_{\text {iron_s }}^{n+1} t_{\mathrm{s}} L_{\mathrm{m}}} \\
& R_{r}^{n}=\frac{0.5\left(r_{\mathrm{ro}}+r_{\mathrm{ri}}\right) 0.5 \theta^{n}}{\mu_{\text {iron_r }}^{n} t_{\mathrm{r}} L_{\mathrm{m}}}+\frac{0.5\left(r_{\mathrm{ro}}+r_{\mathrm{ri}}\right) 0.5 \theta^{n+1}}{\mu_{\text {iro__r }}^{n+1} t_{\mathrm{r}} L_{\mathrm{m}}}
\end{aligned}
$$

where $\mu_{\text {iron_s }}^{n}$ is the relative permeability of $n$th divided sector of stator, $\mu_{\text {iron_r }}^{n}$ is the relative permeability of $n$th divided sector of rotor, $\theta^{n}$ is the angle of $n$th divide sector. This angle $\theta^{n}$ is decided by the design parameters and the modeling parameters $N_{m}, N_{c}$ and $N_{e}$. For example, in Fig. 4, when the $N_{e}=1$, then the $\theta^{0}=2 \alpha_{0}$. There is leakage flux between two poles, and the relevant reluctance is presented in Eq. (4).

$$
R_{m e}^{n}=\frac{0.5\left(r_{m}+r_{r o}\right) 0.5\left(\theta^{n}+\theta^{n+1}\right)}{\mu_{\mathrm{pm}} t_{\mathrm{m}} L_{\mathrm{m}}},
$$

where $\mu_{\mathrm{pm}}$ is relative permeability of PM.

The reluctance in radial direction between neighbor nodes include $R_{g}$ and $R_{m}$ can be represented, respectively, as

$$
\begin{gathered}
R_{\mathrm{g}}^{n}=\frac{t_{\mathrm{c}}+t_{\mathrm{g}}}{\mu_{\text {air }}\left(r_{m}+0.5\left(t_{\mathrm{c}}+t_{\mathrm{g}}\right)\right) \theta^{n} L_{\mathrm{m}}}+\frac{0.5 t_{\mathrm{s}}}{\mu_{\text {iron_s }}^{n} L_{\mathrm{m}} \theta^{n} r_{\mathrm{si}}} \\
R_{\mathrm{m}}^{n}=\frac{t_{\mathrm{m}}}{\mu_{\mathrm{pm}}\left(r_{\text {ro }}+0.5 t_{\mathrm{m}}\right) \theta^{n} L_{\mathrm{m}}}+\frac{0.5 t_{r}}{\mu_{\text {iron_r }} L_{\mathrm{m}} \theta^{n} r_{\text {ro }}}
\end{gathered}
$$

where $\mu_{\text {air }}$ is relative permeability of air. In the present study, $\mu_{\mathrm{pm}}=\mu_{\mathrm{air}}=\mu_{0}$ is assumed.

The equivalent flux source produced from the PMs $\phi_{m}^{n}$ is transformed from magneto-motive source by $\mathrm{KCL} / \mathrm{KVL}$ method which can be express as

$$
\phi_{\mathrm{m}}^{n}=\frac{H_{c} t_{\mathrm{m}}^{n}}{R_{\mathrm{m}}^{n}},
$$

where $H_{c}$ is coercive force of PM, $t_{\mathrm{m}}^{n}$ the thickness of PM. The flux generated by winding current can also be transferred to current source which is given by

$$
\phi_{\mathrm{c}}^{n}=\frac{N_{t}^{n} I}{R_{s}^{n}}
$$

where $N_{t}^{n}$ is the winding turns in the $n$th sector.

In this paper, the EMCM is expressed in matrix form which can be readily solved by KCL method. First, all of the reluctances in Fig. 4 are arranged in a matrix of size $(3 N-1) \times(3 N-1)$, defined as reluctance matrix $\mathbf{G}$ (the size of the matrix is a function of $N$ ), presented as

$$
G=\left[\begin{array}{ccc}
G_{s s} & G_{s m} & 0 \\
G_{m s} & G_{m m} & G_{m r} \\
0 & G_{r m} & G_{r r}
\end{array}\right]_{(3 N-1) \times(3 N-1)}
$$

The details of the sub-matrices $G_{s s}$ to $G_{r r}$ contained in matrix $\mathbf{G}$ are given in Appendix. It is noted that, because the node of rotor which local in middle of one PM is selected as the reference ( $R_{r}^{3}$ in Fig. 4), the size of sub-matrices $G_{r r}, G_{m r}$ and $G_{r m}$ are $(N-1) \times(N-1), N \times(N-1)$ and $(N-1) \times N$, respectively..

The vector of MMF is given as

$$
F=\left[\begin{array}{l}
\left(F_{s}^{n}\right)_{N \times 1} \\
\left(F_{m}^{n}\right)_{N \times 1} \\
\left(F_{r}^{n}\right)_{(N-1) \times 1}
\end{array}\right]_{(3 N-1) \times 1}
$$

The vector of magnetic flux into the node is expressed as

$$
\phi=\left[\begin{array}{l}
\left(\phi_{s}^{n}\right)_{N \times 1} \\
\left(\phi_{m}^{n}\right)_{N \times 1} \\
\left(\phi_{r}^{n}\right)_{(N-1) \times 1}
\end{array}\right]_{(3 N-1) \times 1}
$$

where $\phi_{s}^{n}=\phi_{c}^{n}, \phi_{r}^{n}=0$. Finally, the KCL equation in matrix form is given as

$$
\mathbf{G F}=\phi
$$

thus, the vector of $\mathbf{F}$ can be obtained using the following equation:

$$
\mathbf{F}=\mathbf{G}^{-1} \phi
$$


where $\mathbf{G}^{-1}$ is the inverse of matrix $\mathbf{G}$ in Eq. (12). Then the flux through each sector between neighbor nodes can be calculated. Various flux densities should be identified in motor design, which include flux densities in stator, rotor and airgap. The flux through the $n$th sector of stator and rotor can be expressed as:

$$
\begin{array}{cc}
\phi_{s}^{n}=\frac{F_{s}^{(n+1) \% N}-F_{s}^{n}}{R_{s}^{n}} \quad n=0,1, \cdots, N-1, \\
\phi_{r}^{n}=\frac{F_{r}^{(n+1) \% N}-F_{r}^{n}}{R_{r}^{n}} \quad n=0,1, \cdots, N-1 .
\end{array}
$$

The flux through each sector of air-gap can be represented as

$$
\phi_{g}^{n}=\frac{F_{m}^{n}-F_{s}^{n}}{R_{g}}
$$

Then, the flux density of each sector can be calculated by the division of flux by the area.

It should be noted that, replacing voltage sources with current source and in parallel with an equivalent resistance in the KCL method can keep the same results of the node voltage but cannot keep the same flux through the resistance in parallel. Therefore, when calculating flux density in stator, the excitation current should be set to 0 , i.e., let $\phi_{s}^{n}=0$. Then calculated flux density is only contributed by PM. Since the flux generated by current is usually much smaller than by the PM, this assumption will not influence the results significantly. The excitation current can be set when calculating flux density in the air-gap and output torque.

An equivalent method in which the rotor is fixed in position and the coil is assumed sliding along the stator is adopted to calculate the flux at a certain rotor angle. Under this assumption, the rotation of rotor can be simply achieved by updating the flux generated by the coil in each sector. This method employs the fixed network and will not affect the correctness of the results since the stator is circumferentially symmetric.

In the proposed EMCM, the magnetic circuit networks are treated as a function of $N_{p}$, and the modeling accuracy can be defined by $N_{e}, N_{c}$ and $N_{m}$. Therefore, the approach can be easily adjusted to different pole number and precision. There is no need to rebuild the network. It is a general modeling approach for toroidally-wound limited angle servo motors.

\section{B. Nonlinear Material Considerations}

The stator and rotor are made by soft iron material with nonlinear B-H relationship. The relationship between relative permeability and magnetic flux density in soft iron can be modeled by the function below as [6]:

$$
\mu=\frac{B}{\left(k_{1} e^{k_{2} B^{2}}+k_{3}\right)}
$$

where $k_{1}, k_{2}$ and $k_{3}$ are constant for different materials. For example, the constants of annealed steel is $k_{1}=2.6, k_{2}=$ 2.72 and $k_{3}=154.4$. The $\mu$-B curve is shown in Fig. 5 .

In order to improve the accuracy of calculation, it is necessary for reluctance of $R_{s}^{n}$ and $R_{r}^{n}$ to be updated by an iterative process. First, an reasonable permeability value,



Fig. 5. Relative permeability curve vs flux density of steel.

such as 4000 , is given to each sector of stator and rotor to initiate the calculation. After the first round of calculation, the flux density of each sector can be derived by Eq. (17). Then the new relative permeability of each sector can be iteratively calculated by

$$
\mu_{k+1}^{n}=\frac{B_{k}^{n}}{\left(k_{1} e^{\left.k_{2}\left(B_{k}^{n}\right)^{2}+k_{3}\right)}\right.}
$$

Second, the updated $\mu_{s / r, k+1}^{n}$ is fed back to the model to replace the last value of the component. Finally, more iteration is usually required to help the process convergence. The stop condition can be restricted by the variation of the permeability during last two iterations.

$$
\max \left[\left|\mu_{k+1}^{n}-\mu_{k}^{n}\right|\right]<\epsilon \quad n=0,1, \cdots, N-1
$$

where $\epsilon$ is a small positive constant.

In order to increase the stability of the iterating process, an inertia term $\alpha$ is introduced, and the replacement approach is presented as:

$$
\mu_{k+1}^{n}=\alpha \mu_{k}^{n}+(1-\alpha) \mu_{k+1}^{n}
$$

\section{VERIFICATION OF EMCM B Y FEM}

The EMEC model should be verified before utilized for optimization. In the present study, this model is verified by finite element analysis (FEA) method. The design parameters of LMSM for comparison with FEA and EMEC model are listed in Table. I. Two group design parameters are adopted.

TABLE I

VERIFICATION DESIGN PARAMETERS

\begin{tabular}{llrr}
\hline Parameters (Units) & Symbols & Design 1 & Design 2 \\
\hline Inner radius of rotor $(\mathrm{mm})$ & $r_{r i}$ & 15.5 & 15.5 \\
Thickness of rotor $(\mathrm{mm})$ & $t_{r}$ & 8 & 9 \\
Thickness of PM (mm) & $t_{m}$ & 8 & 6 \\
Thickness of air-gap (mm) & $t_{g}$ & 0.5 & 1 \\
Thickness of stator $(\mathrm{mm})$ & $t_{s}$ & 8 & 8.5 \\
Diameters of conductor $(\mathrm{mm})$ & $d_{c}$ & 0.5 & 0.5 \\
Winding Layers & $L_{w}$ & 4 & 4 \\
Axis length (mm) & $L_{\mathrm{m}}$ & 25 & 25 \\
Angle gap (deg) & $\alpha_{0}$ & 2 & 2 \\
Constant angle range $(\mathrm{deg})$ & $\alpha_{1}$ & 12 & 12 \\
\hline
\end{tabular}

The flux density and flux lines distribution of the 4-pole LASM by FEA is shown in Fig. 6. In order to compare with the EMCM model, three lines, which are located in the middle of the rotor, air-gap and stator, are added in the FEA model.

The flux density in the air-gap by FEA and EMCM is shown in Fig. 7. The flux density in the stator is shown in Fig. 8. The flux density in the rotor is shown in Fig. 9, and there are two sets of results by EMCM in different design parameters. The first design has two different conditions which uses different 


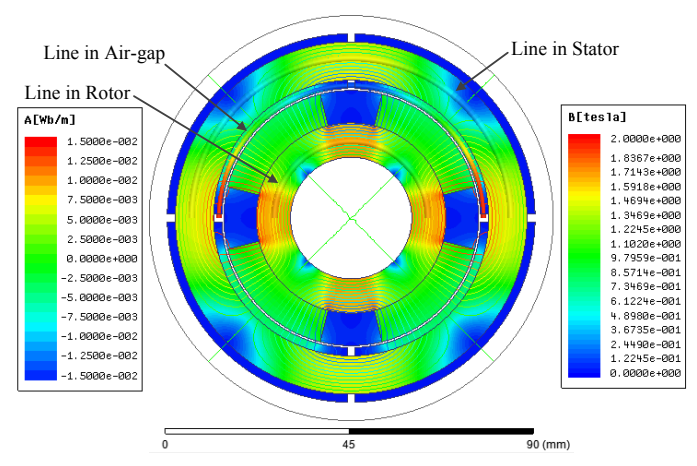

Fig. 6. FEA results of flux density and flux line distribution

calculation points. The results indicate that the results of EMCM agree with FEM in different design parameters, and the accuracy of the results increases with the sector number. The flux density results of the air-gap and the stator by EMCM are rather close to the FEA results. The flux density results of rotor by EMCM agree well with the FEA results at the peak value, but trend to deviate rather significantly between the peaks. This can be explained as the results of FEA are only on the selected line which only indicate the middle points of the sector. But the results of EMCM are the average flux density determined by the total flux divided by the section area. When flux density is small, the flux will not distribute evenly, as shown in Fig. 6. That explains why the small values do not agree well. The flux trend to distribute more evenly when the flux density is close to saturation value. That explains the good agreements at the peaks. Fortunately, normally only the maximum flux densities in stator and rotor are of interest for the avoidance of saturation in motor design.



Fig. 7. Comparison of flux density in air-gap of EMCM and FEA

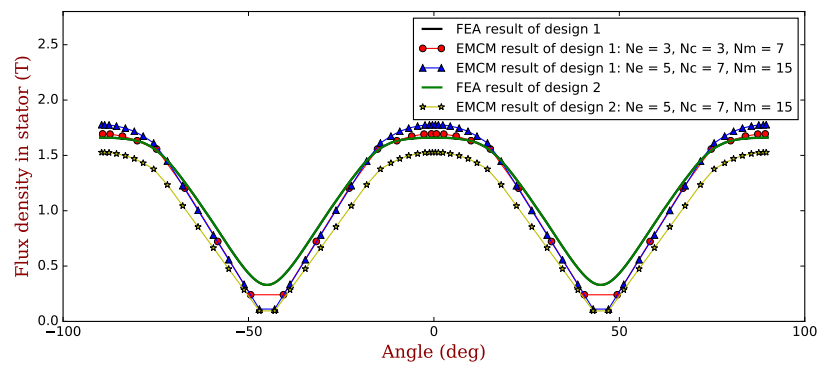

Fig. 8. Comparison of flux density in stator of EMCM and FEA

The torques with different excitation currents and angular positions obtained by FEA and EMCM model are shown in Fig. 10. The two dimension torque error distribution between these two methods are shown in Fig. 11. These two figures indicate that the torque is linearly proportional to the current, and it is almost constant in the range of $\pm 10^{\circ}$. The difference

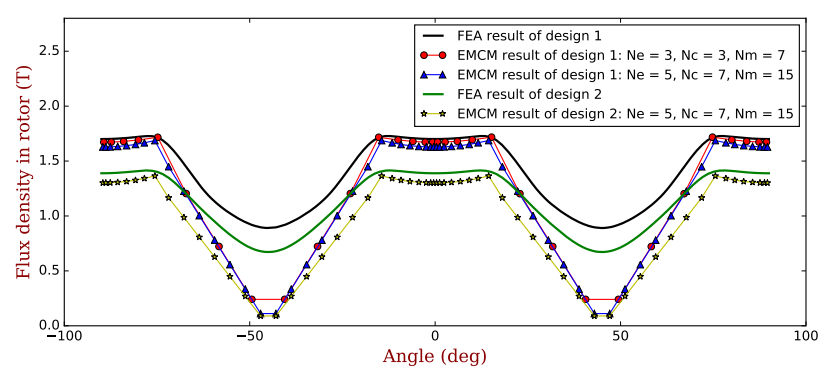

Fig. 9. Comparison of flux density in rotor of EMCM and FEA

between FEA and EMCM method are within 5\%.
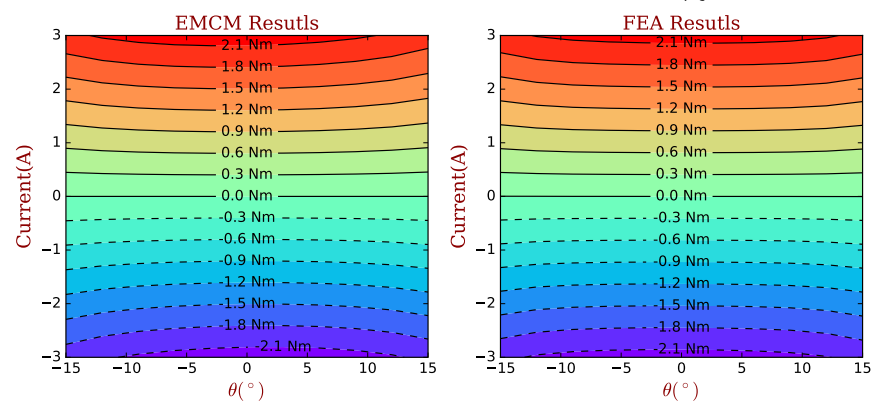

Fig. 10. Torque versus current and position by EMCM and FEA (design 1)



Fig. 11. The error of torque obtained by EMCM and FEA (design 1)

The comparison of flux density and electromagnetic torque between the FEA and EMCM methods indicate that the accuracy of EMCM is acceptable for preliminary design. Moreover, the calculation time for FEA is over minutes while the EMCM only needs $0.1 s$ when $N_{e}=5, N_{c}=7$ and $N_{m}=15$. Thus, the EMCM is particularly suitable for preliminary design for much shorter computation time.

\section{Multi-objective Optimization}

\section{A. Optimization Parameters}

The process of preliminary design is to get a set of optimized design parameters according to the given requirements. Usually, the given requirements include the torque constant $K_{t}$, angle $\alpha_{1}$ which is the rotation range of the LASM, and the clearance between the winding and PM $t_{g}$ for avoidance of contact. There are 8 parameters that are supposed to be decided during the preliminary design phase which are listed below.

- Poles of design motor $N_{p}$. 
- Inner radius of rotor $r_{r i}$.

- Thickness of PM $t_{m}$.

- Thickness of rotor $t_{r}$.

- Thickness of stator $t_{s}$.

- The diameter of conductor $d_{c}$.

- The layers of conductor $L_{w}$.

- Axis length of motor $L_{m}$.

The pole numbers $N_{p}$ should conform to the desired angular range $\alpha_{1}$. If $\alpha_{1}$ is big, the $N_{p}$ should be small since the empty angle is $N_{p} \alpha_{1}$ which would degrade the torque constant. When $\alpha_{1}$ is small, bigger $N_{p}$ means the magnetic circuit between two neighbor poles is shorter and needs less iron to avoid saturation. Then the thickness of iron can be reduced and allows thicker PM within the same space which positive for torque constant. A proper radius of $r_{r i}$ can reduce the mass and inertia of rotor without saturation. The $t_{m}$ is the primary determinant of the air gap flux density. Increase $t_{m}$ could enlarge the air-gap flux density but the increase in gradient gets smaller. And the mass and inertia are increasing linearly and squarely respectively with $t_{m}$. Thus, $t_{m}$ should be optimized for maximum overall performance. Both $t_{r}$ and $t_{s}$ influence the flux density in the rotor and stator. They should be optimized to make the flux density close to the saturation value to maximize material efficiency. The $d_{c}$ and $L_{w}$ determine the resistance and inductance of the winding respectively. The thickness of winding $t_{c}$ equals $L_{w} \times d_{c}$.

\section{B. Optimization Objectives}

Usually, there are more than one objectives that should be considered for a LASM design. The objectives of the LASM considered in the present study are listed below.

- Torque constant requirement $K_{t}$. The $K_{t}$ is the most important requirement which is decided by the application. It is an expected value which must be satisfied. For the LASM, the $K_{t}=B_{g} L_{m} N_{c} r_{g}$, where $B_{g}$ is the magnetic flux density of air gap which is influenced by design parameters of $r_{r i}, t_{r}, t_{m}, t_{g}, t_{c}$ and $\alpha_{1}$, another parameter $N_{c}$ is the winding turns which is decided by $r_{r i}, t_{r}, t_{m}$, $t_{g}, d_{c}$ and $L_{w}$.

- Winding resistance. Winding resistance is influenced by $d_{c}, L_{m}, L_{w}$ and $t_{s}$. For the same winding turns, use of smaller conductor can reduce $t_{c}$ which can enhance air-gap flux density but will increase winding resistance. Bigger resistance means bigger copper losses which reduce the efficiency and generate more heat which in turn will increase temperature and reduce reliability. It also requires higher input voltage to generate the same current. The input voltage in some applications is limited. For example, the aircraft can only offer $28 \mathrm{~V}_{\mathrm{DC}}$. On the contrary, smaller resistance needs bigger conductor which will increase $t_{c}$ which reduce air-gap flux density, and also increase the size and the mass of the motor.

- Dynamic response time delay. The dynamic response is important for servo application. The simplified transfer function between applied voltage, $U$, and the motor shaft speed, $\omega$, can be presented as $\omega(s) / U(s)=K /\left(\tau^{2} s^{2}+\right.$ $2 \zeta \tau s+1)$, where the steady-stage gain $K=1 / K_{e}\left(K_{e}\right.$ is the back-emf coefficient). It is a second order system. The characteristic time is $\tau=\sqrt{(L J) /\left(K_{t} K_{e}\right)}$, and the damping ratio $\zeta=(R / 2 L) \tau$. The dynamic performance should be assessed under two conditions that is distinguished by $\zeta$. The first condition is overdamped $(\zeta>1)$. Then the second order system can be regarded as two first order terms series as $1 /\left(\left(\tau_{1} s+1\right)\left(\tau_{2} s+1\right)\right)$, where $\tau_{1,2}=\tau /\left(\zeta \pm \sqrt{\zeta^{2}-1}\right)$ are two effective time constants. The total time delay can be presented as $\tau_{\mathrm{t}}=\tau_{1}+\tau_{2}$. The second condition is under damped $(\zeta<1)$. The time constant usually can be defined as the real part of the roots of the characteristic equations, $\tau / \zeta$. But, increasing the imaginary part also can shorten the response time but introduces overshoot. Nonetheless, if the damping ratio is limited within a acceptable range, for example, $0.7<\zeta<1$, the quicker response is preferred for servo application. Therefore, in this condition, the total time delay can be defined as the inverse of the sum of the norm length of vector of the two poles, which equals to $\tau_{t}=2 \tau$. The $\zeta \leq 0.7$ is limited in present study for stability. In summary, the definition of the total time delay is given as

$$
\tau_{t}= \begin{cases}\frac{\tau}{\zeta-\sqrt{\zeta^{2}-1}}+\frac{\tau}{\zeta+\sqrt{\zeta^{2}-1}} & \zeta \geq 1 \\ 2 \tau & 1>\zeta>0.7 \\ \infty & 0.7 \leq \zeta\end{cases}
$$

- Mass of motor. The mass of motor is important for some special applications such as in aerospace.

- Maximum iron material utilization efficiency. The maximum magnetic flux density in the stator and rotor should be close to the "knee value" of B-H curve of the material.

\section{Optimization Objective Function}

An objective function should be formulated mathematically for the optimization process. This function should take into account all aspects to be optimized. The value of this function is usually called the "fitness value" which can be used to assess the overall performance.

The optimization objectives listed in the last sub-section can be classified into three different types and utilize different fitness functions. The first type is that an expected value should be definitely satisfied, bigger or smaller. For example, the torque constant has to be bigger than the application requirement; the winding resistance should be smaller than a value for drive system requirement. For this type of objective, an anti-tangent function is utilized as shown in Eq. (22) and the curve is shown in Fig. 12. If the $x$ is bigger than $x_{\text {req, }}$, $J(x) \rightarrow 1$, other way, the $J(x) \rightarrow 0$. The coefficient $W$ can adjust the slope between 0 and 1 .

$$
J(x)=0.5+\frac{1}{\pi} \arctan \left(W\left(x-x_{\text {req }}\right)\right)
$$

The second type is one that the objective value should be close to a fixed value. One example is the maximum flux density of the iron core. For optimized design, the maximum flux density should be close to the saturation point which is 


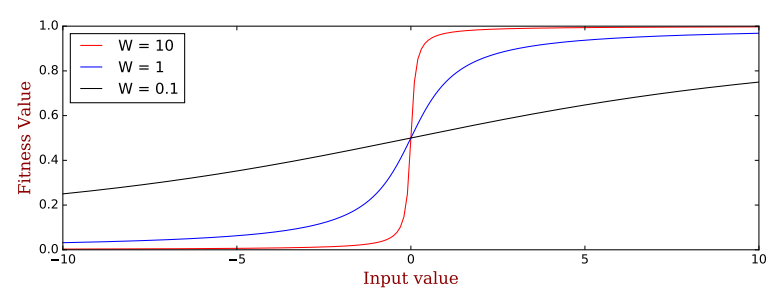

Fig. 12. The curve of requirement type objective fitness function

the "knee point" in the B-H curve of the magnetism material. If the maximum flux density in the iron is less than that value, it means the weight is not optimized and implies material wastage. On the contrary, bigger than saturation value means magnetic energy is wasted. This kind of objective is evaluated by the reciprocal of a square function which is presented in Eq. (23) and the curve is shown in Fig. 13. If $x$ is closed to the $x_{\text {fix }}, J(x)$ trends to 1 , far from the value in both side, $J(x) \rightarrow 0$. The coefficient $W$ is used to adjust the interval width.

$$
J(x)=\frac{1}{\left(W\left(x-x_{\mathrm{fix}}\right)\right)^{2}+1}
$$

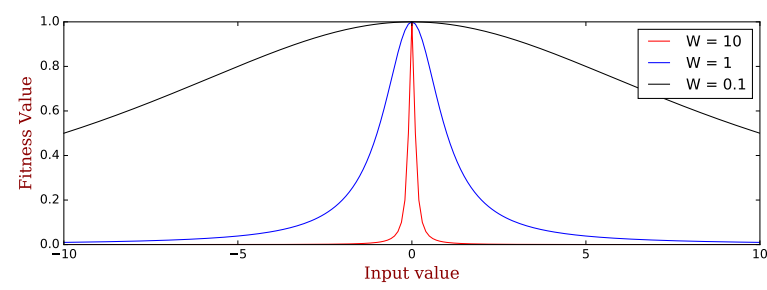

Fig. 13. The curve of fixed value type objective fitness function

The third type is one that has only the expected trend of the objective. For example, less mass is usually better, shorter time constant means quick response and suitable for servo performance. This kind objective is evaluated by a proportional function which is presented in Eq. (24). $x_{\text {ref }}$ is a reference value to normalize the fitness value.

$$
J(x)=W f\left(x, x_{\text {ref }}\right)
$$

The final optimization objective function is defined as Eq. (25). The first and second type objectives are treated as product factor which should be satisfied, and the third type objectives are summed up as one product factor.

$$
\begin{aligned}
J & =J_{1} J_{2} J_{3} J_{4}\left(J_{5}+J_{6}\right) \\
J_{1} & =\frac{1}{\left(W_{1}\left(B_{s}-B_{\max }\right)\right)^{2}+1} \\
J_{2} & =\frac{1}{\left(W_{2}\left(B_{r}-B_{\max }\right)\right)^{2}+1} \\
J_{3} & =0.5+\frac{1}{\pi} \arctan \left(W_{3}\left(K_{t}-K_{t, \text { req }}\right)\right) \\
J_{4} & =0.5+\frac{1}{\pi} \arctan \left(W_{4}\left(R_{\max }-R\right)\right) \\
J_{5} & =W_{5} \frac{m_{\text {ref }}}{m}, \quad J_{6}=W_{6} \frac{\tau_{\text {t, ref }}}{\tau_{\mathrm{t}}}
\end{aligned}
$$

The optimization is carried out by the widely used particle swarm optimization (PSO) method [13-16]. In PSO, a swarm of particles are represented as potential solutions, and each particle $i$ is associated with two vectors, i.e., the velocity vector $\mathbf{V}_{i}=\left[v_{i}^{1}, v_{i}^{2}, \ldots, v_{i}^{D}\right]$, and the position vector $\mathbf{X}_{i}=$ $\left[x_{i}^{1}, x_{i}^{2}, \ldots, x_{i}^{D}\right]$, where $D$ represents the dimensions of the solution space. During the evolutionary process of PSO, the particles are first initialized randomly in the solution space. Then, the particles "fly" to search the global optimization position under the searching law as follow:

$$
\begin{aligned}
v_{i}^{d}= & w v_{i}^{d}+c_{1} \operatorname{rand}()_{i}^{d}\left(\operatorname{pBest}_{i}^{d}-x_{i}^{d}\right) \\
& +c_{2} \operatorname{rand}()_{i}^{d}\left(\operatorname{gBest}^{d}-x_{i}^{d}\right) \\
x_{i}^{d}= & x_{i}^{d}+v_{i}^{d}
\end{aligned}
$$

where $w$ is the inertia weight, $c_{1}$ and $c_{2}$ are the acceleration coefficients, and $\operatorname{rand}()_{i}^{d}$ is a uniformly distributed random number within $[0,1]$, the superscript $d$ is abbreviation of "dimension", $\mathrm{pBest}_{i}^{d}$ means the $d$-th position of where get the best fitness value searched by the $i$-th particle, and the gBest ${ }^{d}$ presents the $d$-th position of where get the global best fitness value searched by all particles.

\section{Optimization results and discussion}

The optimization process is based on the EMCM model. The optimization parameters and bound are shown in Tab. II.

TABLE II

OPTIMIZATION PARAMETERS AND BOUND

\begin{tabular}{lrr}
\hline Optimization parameters (Units) & Lower bound & Upper bound \\
\hline$r_{r i}(\mathrm{~mm})$ & 0 & 20 \\
$t_{r}(\mathrm{~mm})$ & 3 & 15 \\
$t_{m}(\mathrm{~mm})$ & 2 & 15 \\
$t_{s}(\mathrm{~mm})$ & 3 & 15 \\
$L_{m}(\mathrm{~mm})$ & 15 & 60 \\
$d_{c}(\mathrm{~mm})$ & 0.2 & 1 \\
$L_{w}$ (layers) & 2 & 6 \\
$N_{p}$ (poles) & 2 & 8 \\
\hline
\end{tabular}

Six different cases are studied for comparison. The required angular range of Case $1 \& 2$, Case $3 \& 4$, Case $5 \& 6$ are $10^{\circ}, 20^{\circ}$, and $30^{\circ}$, respectively. Cases 1,3 and 5 have higher weight coefficient on mass while cases 2,4 and 6 have higher weight coefficient on time constant. In the present study, the parameters of the PSO method include: particles number $=250$, inertia factor $w=0.5$. The particle memory influence $c_{1}=1.5$, and the swarm influence $c_{2}=1.5$. The optimization time consumption is about $1500 \mathrm{~s}$ for each case. The parameters and optimization results of these six cases are shown in Table. III.

The angular range is the major decision factor of pole numbers. The optimized pole number of case 1 and 2 is 5 or 6 , while 4 for case 3 and 4,2 or 3 for case 5 and 6 . This is because with the increasing of $\alpha_{1}$, the bigger pole number means more empty magnetized sector of rotor, which will result in poor output torque. The optimization process can find the most suitable pole number for the desired angular range.

The trade off between mass $\left(J_{5)}\right)$ and time constant $\left(J_{6}\right)$ results in different motor structures. Usually, for a required torque constant, a disk like design with short axis length and large radius is less mass but long response time. On the contrary, a bar-like design with long axial length and small 
radius will lead to short response time but bigger mass. The first kind is usually called torque motor while the second type is named servo motor. The optimization results conform to these design rules. The optimized shape of case 1, 3 and 5 which focus on weight optimization are stubby, while the shape of case 2, 4, and 6 are spindly which focus on time constant. All of the three cases of 2, 4, and 6 reach the bound of the length.

The first and second type objectives $\left(J_{1}\right.$ to $\left.J_{4}\right)$ are all satisfied in each case. The maximum flux density of stator and rotor for each case are close to the setting value 1.6T. The $K_{t}$ 's are all bigger than $0.5 \mathrm{Nm}$ and $R$ 's are all smaller than $9 \Omega$. These results comfirm the effectiveness of proposed fitness functions.

\section{CONCLUSIONS}

An EMCM has been proposed for the preliminary design and optimization of a LASM. A generalized model of toroidally-wound type LASM has been constructed as a function of design parameters. The model was arranged in KCL matrix format which is easy to be extended and solved. An iterating technique to obtain accurate permeability of the nonlinear magnetic material is also incorporated into the model. The accuracy of the proposed model has been verified by FEA method in two typical designs. A multi-objective optimization method of the same type of LASM is developed based on the verified EMCM model. A multi-objective fitness function using three kinds of objective functions is proposed. Six different requirement cases are optimized by the proposed method. The optimization results illustrate the feasibility of the method. It is shown that the proposed generalized EMCM model is sufficiently accurate for the preliminary design and optimization of a LASM at minimum computational cost. It is envisaged that the generalized model can be applied to other electromagnetic devices.

\section{APPENDIX A}

Details of SUbMATRICES IN EQ. (9)

The sub-matrices in matrix $\mathbf{G}$ are given in the following.

$$
\begin{aligned}
& G_{s s}= {\left[\begin{array}{ccccc}
G_{s s}^{11} & \frac{-1}{R_{s}^{0}} & 0 & \cdots & \frac{-1}{R_{s}^{N-1}} \\
\frac{-1}{R_{s}^{0}} & G_{s s}^{22} & \frac{-1}{R_{s}^{1}} & \cdots & 0 \\
\vdots & & & \ddots & \frac{-1}{R_{s}^{N-2}} \\
\frac{-1}{R_{s}^{N-1}} & \cdots & 0 & \frac{-1}{R_{s}^{N-2}} & G_{s s}^{N N}
\end{array}\right]_{N \times N} } \\
& G_{s s}^{11}= \frac{1}{R_{s}^{0}}+\frac{1}{R_{s m}^{0}}+\frac{1}{R_{s}^{N-1}}, G_{s s}^{22}=\frac{1}{R_{s}^{0}}+\frac{1}{R_{s m}^{1}}+\frac{1}{R_{s}^{1}} \\
& G_{s s}^{N N}=\frac{1}{R_{s}^{N-1}}+\frac{1}{R_{s m}^{N-1}}+\frac{1}{R_{s}^{N-2}} \\
& G_{m m}=\left[\begin{array}{ccccc}
G_{m m}^{11} & \frac{-1}{R_{m e}^{0}} & 0 & \cdots & \frac{-1}{R_{m e}^{N-1}} \\
\frac{-1}{R_{m e}^{0}} & G_{m m}^{22} & \frac{-1}{R_{m e}^{2}} & \cdots & 0 \\
\vdots & & & \ddots & \frac{-1}{R_{m}^{N-2}} \\
\frac{-1}{R_{m e}^{N-1}} & \cdots & 0 & \frac{-1}{R_{m e}^{N-2}} & G_{m m}^{N N}
\end{array}\right]_{N \times N}
\end{aligned}
$$

$$
\begin{aligned}
& G_{m m}^{11}=\frac{1}{R_{m e}^{0}}+\frac{1}{R_{g}^{0}}+\frac{1}{R_{m}^{0}}+\frac{1}{R_{m e}^{N-1}} \\
& G_{m m}^{22}=\frac{1}{R_{m e}^{1}}+\frac{1}{R_{g}^{1}}+\frac{1}{R_{m}^{1}}+\frac{1}{R_{m e}^{2}} \\
& G_{m m}^{N N}=\frac{1}{R_{m e}^{N-1}}+\frac{1}{R_{g}^{N-1}}+\frac{1}{R_{m}^{N-1}}+\frac{1}{R_{m e}^{N-2}} \\
& G_{r r}=\left[\begin{array}{ccccc}
G_{r r}^{11} & \frac{-1}{R_{r}^{0}} & 0 & \cdots & \frac{-1}{R_{r}^{N-1}} \\
\frac{-1}{R_{r}^{0}} & G_{r r}^{22} & \frac{-1}{R_{r}^{2}} & \cdots & 0 \\
\vdots & & & \ddots & \frac{-1}{R_{r}^{N-2}} \\
\frac{-1}{R_{r}^{N-1}} & \cdots & 0 & \frac{-1}{R_{r}^{N-2}} & G_{r r}^{(N-1)^{2}}
\end{array}\right]_{(N-1)^{2}} \\
& G_{r r}^{11}=\frac{1}{R_{r}^{0}}+\frac{1}{R_{m}^{0}}+\frac{1}{R_{r}^{N-1}}, G_{r r}^{22}=\frac{1}{R_{r}^{1}}+\frac{1}{R_{m}^{1}}+\frac{1}{R_{r}^{2}} \\
& G_{r r}^{(N-1)^{2}}=\frac{1}{R_{r}^{N-1}}+\frac{1}{R_{m}^{N-1}}+\frac{1}{R_{r}^{N-2}} \\
& G_{s m}=G_{m s}^{T}=\left[\begin{array}{cccc}
\frac{-1}{R_{g}^{0}} & 0 & \cdots & 0 \\
0 & \frac{-1}{R_{g}^{1}} & \cdots & 0 \\
\vdots & \cdots & \ddots & 0 \\
0 & 0 & \cdots & \frac{-1}{R_{g}^{N-1}}
\end{array}\right]_{N \times N} \\
& G_{r m}=G_{m r}^{T}=\left[\begin{array}{cccc}
\frac{-1}{R_{m}^{0}} & 0 & \cdots & 0 \\
0 & \frac{-1}{R_{m}^{1}} & \cdots & 0 \\
\vdots & \cdots & \ddots & 0 \\
0 & 0 & \cdots & \frac{-1}{R_{m}^{N-1}}
\end{array}\right]_{(N-1) \times N}
\end{aligned}
$$

\section{ACKNOWLEDGMENT}

This work is supported by National Aviation Science Foundation (No. 20130751013) and National Key Basic Research and Development Program (2014CB046401). Chinese International Science and Technology Cooperation Program (Project No. 2011DFA72690). This work is supported partially by Chinese Scholar Council, and Cranfield University who is host to the first author.

\section{REFERENCES}

[1] C. Dawson and H. Bolton, "Limited motion rotary actuators of the toroidal-stator, permanent-magnet rotor type," in IEE Proceedings B (Electric Power Applications), vol. 129, no. 4. IET, 1982, pp. 190-198.

[2] K. Chau, M. Cheng, and C. Chan, "Nonlinear magnetic circuit analysis for a novel stator doubly fed doubly salient machine," Magnetics, IEEE Transactions on, vol. 38, no. 5, pp. 2382-2384, 2002.

[3] H. Polinder, J. G. Slootweg, M. J. Hoeijmakers, and J. C. Compter, "Modeling of a linear pm machine including magnetic saturation and end effects: Maximum force-tocurrent ratio," Industry Applications, IEEE Transactions on, vol. 39, no. 6, pp. 1681-1688, 2003.

[4] C. Pompermaier, K. Kalluf, A. Zambonetti, M. Luz, and I. Boldea, "Small linear pm oscillatory motor: magnetic circuit modeling corrected by axisymmetric 2-d fem 
TABLE III

OPTIMIZATION RESULTS OF DIFFERENT WEIGHT OF FITNESS VALUES

\begin{tabular}{|c|c|c|c|c|c|c|}
\hline Angular range & Weight Coe. & Parameters & Evaluation Results & Weight Coe. & Parameters & Evaluation Results \\
\hline $\begin{array}{l}\text { Case } 1 \text { and 2: } \\
\alpha_{0}=2^{\circ} \\
\alpha_{1}=10^{\circ}\end{array}$ & $\begin{array}{l}W_{1}=10 \\
W_{2}=10 \\
W_{3}=100 \\
W_{4}=100 \\
W_{5}=100 \\
W_{6}=10\end{array}$ & $\begin{array}{l}r_{r i}=15.72 \mathrm{~mm} \\
t_{r}=6.72 \mathrm{~mm} \\
t_{m}=10.14 \mathrm{~mm} \\
t_{s}=6.13 \mathrm{~mm} \\
d_{c}=0.6 \mathrm{~mm} \\
L_{w}=5 \\
L_{m}=20.51 \mathrm{~mm} \\
N_{p}=6\end{array}$ & $\begin{array}{l}m=0.602 \mathrm{~kg} \\
K_{t}=0.608 \mathrm{Nm} / \mathrm{A} \\
R=7.08 \Omega \\
B_{\mathrm{s}, \max }=1.61 \mathrm{~T} \\
B_{\mathrm{r}, \max }=1.60 \mathrm{~T} \\
J=0.375 \mathrm{~g} / \mathrm{m}^{2} \\
\tau_{\mathrm{t}}=7.1 \mathrm{~ms} \\
L=2.4 \mathrm{mH}\end{array}$ & $\begin{array}{l}W_{1}=10 \\
W_{2}=10 \\
W_{3}=100 \\
W_{4}=100 \\
W_{5}=10 \\
W_{6}=100\end{array}$ & $\begin{array}{l}r_{r i}=4.7 \mathrm{~mm} \\
t_{r}=7.65 \mathrm{~mm} \\
t_{m}=10.1 \mathrm{~mm} \\
t_{s}=9.7 \mathrm{~mm} \\
d_{c}=0.7 \mathrm{~mm} \\
L_{w}=4 \\
L_{m}=60 \mathrm{~mm} \\
N_{p}=5\end{array}$ & $\begin{array}{l}m=1.586 \mathrm{~kg} \\
K_{t}=0.835 \mathrm{Nm} / \mathrm{A} \\
R=6.01 \Omega \\
B_{\mathrm{s}, \max }=1.63 \mathrm{~T} \\
B_{\mathrm{r}, \max }=1.60 \mathrm{~T} \\
J=0.29 \mathrm{~g} / \mathrm{m}^{2} \\
\tau_{\mathrm{t}}=2.5 \mathrm{~ms} \\
L=2.8 \mathrm{mH}\end{array}$ \\
\hline $\begin{array}{l}\text { Case } 3 \text { and } 4: \\
\alpha_{0}=2^{\circ} \\
\alpha_{1}=20^{\circ}\end{array}$ & $\begin{array}{l}W_{1}=10 \\
W_{2}=10 \\
W_{3}=100 \\
W_{4}=100 \\
W_{5}=100 \\
W_{6}=10\end{array}$ & $\begin{array}{l}r_{r i}=15.2 \mathrm{~mm} \\
t_{r}=8.6 \mathrm{~mm} \\
t_{m}=11.5 \mathrm{~mm} \\
t_{s}=10.6 \mathrm{~mm} \\
d_{c}=0.9 \mathrm{~mm} \\
L_{w}=6 \\
L_{m}=19.4 \mathrm{~mm} \\
N_{p}=4\end{array}$ & $\begin{array}{l}m=1.11 \mathrm{~kg} \\
K_{t}=0.545 \mathrm{Nm} / \mathrm{A} \\
R=3.69 \Omega \\
B_{\mathrm{s}, \max }=1.60 \mathrm{~T} \\
B_{\mathrm{r}, \max }=1.60 \mathrm{~T} \\
J=0.48 \mathrm{~g} / \mathrm{m}^{2} \\
\tau_{\mathrm{t}}=6.2 \mathrm{~ms} \\
L=5.98 \mathrm{mH} \\
\end{array}$ & $\begin{array}{l}W_{1}=10 \\
W_{2}=10 \\
W_{3}=100 \\
W_{4}=100 \\
W_{5}=10 \\
W_{6}=100\end{array}$ & $\begin{array}{l}r_{r i}=6.6 \mathrm{~mm} \\
t_{r}=7.6 \mathrm{~mm} \\
t_{m}=14.2 \mathrm{~mm} \\
t_{s}=9.1 \mathrm{~mm} \\
d_{c}=0.8 \mathrm{~mm} \\
L_{w}=5 \\
L_{m}=60 \mathrm{~mm} \\
N_{p}=4\end{array}$ & $\begin{array}{l}m=2.0 \mathrm{~kg} \\
K_{t}=1.09 \mathrm{Nm} / \mathrm{A} \\
R=6.59 \Omega \\
B_{\mathrm{s}, \max }=1.62 \mathrm{~T} \\
B_{\mathrm{r}, \max }=1.60 \mathrm{~T} \\
J=0.59 \mathrm{~g} / \mathrm{m}^{2} \\
\tau_{\mathrm{t}}=3.3 \mathrm{~ms} \\
L=5.0 \mathrm{mH}\end{array}$ \\
\hline $\begin{array}{l}\text { Case } 5 \text { and } 6 \text { : } \\
\alpha_{0}=2^{\circ} \\
\alpha_{1}=30^{\circ}\end{array}$ & $\begin{array}{l}W_{1}=10 \\
W_{2}=10 \\
W_{3}=100 \\
W_{4}=100 \\
W_{5}=100 \\
W_{6}=10\end{array}$ & $\begin{array}{l}r_{r i}=11.8 \mathrm{~mm} \\
t_{r}=7.2 \mathrm{~mm} \\
t_{m}=15.0 \mathrm{~mm} \\
t_{s}=9.9 \mathrm{~mm} \\
d_{c}=0.7 \mathrm{~mm} \\
L_{w}=4 \\
L_{m}=30.0 \mathrm{~mm} \\
N_{p}=3\end{array}$ & $\begin{array}{l}m=1.04 \mathrm{~kg} \\
K_{t}=0.62 \mathrm{Nm} / \mathrm{A} \\
R=5.3 \Omega \\
B_{\mathrm{s}, \max }=1.62 \mathrm{~T} \\
B_{\mathrm{r}, \max }=1.61 \mathrm{~T} \\
J=0.48 \mathrm{~g} / \mathrm{m}^{2} \\
\tau_{\mathrm{t}}=6.7 \mathrm{~ms} \\
L=5.9 \mathrm{mH}\end{array}$ & $\begin{array}{l}W_{1}=10 \\
W_{2}=10 \\
W_{3}=100 \\
W_{4}=100 \\
W_{5}=10 \\
W_{6}=100\end{array}$ & $\begin{array}{l}r_{r i}=1.7 \mathrm{~mm} \\
t_{r}=7.5 \mathrm{~mm} \\
t_{m}=10.3 \mathrm{~mm} \\
t_{s}=10.7 \mathrm{~mm} \\
d_{c}=0.6 \mathrm{~mm} \\
L_{w}=4 \\
L_{m}=60.0 \mathrm{~mm} \\
N_{p}=2\end{array}$ & $\begin{array}{l}m=1.32 \mathrm{~kg} \\
K_{t}=0.62 \mathrm{Nm} / \mathrm{A} \\
R=8.42 \Omega \\
B_{\mathrm{s}, \max }=1.63 \mathrm{~T} \\
B_{\mathrm{r}, \max }=1.60 \mathrm{~T} \\
J=0.135 \mathrm{~g} / \mathrm{m}^{2} \\
\tau_{\mathrm{t}}=2.9 \mathrm{~ms} \\
L=6.1 \mathrm{mH}\end{array}$ \\
\hline
\end{tabular}

and experimental characterization," Industrial Electronics, IEEE Transactions on, vol. 59, no. 3, pp. 1389-1396, 2012.

[5] Y. Liu, M. Zhang, Y. Zhu, J. Yang, and B. Chen, "Optimization of voice coil motor to enhance dynamic response based on an improved magnetic equivalent circuit model," Magnetics, IEEE Transactions on, vol. 47, no. 9, pp. 2247-2251, 2011.

[6] J. R. Brauer, Magnetic actuators and sensors. John Wiley \& Sons, 2006.

[7] M.-F. Hsieh and Y.-C. Hsu, "A generalized magnetic circuit modeling approach for design of surface permanentmagnet machines," Industrial Electronics, IEEE Transactions on, vol. 59, no. 2, pp. 779-792, 2012.

[8] R. N. Zarandi, H. MeshginKelk, F. Toorani, and H. Farahmandzad, "Comprehensive design of a toroidally-wound limited angle torque motor," Journal of International Review of Electrical Engineering (IREE), vol. 6, pp. 198206, 2011.

[9] M. Roohnavazfar, M. Houshmand, R. N. Zarandi, and M. Mirsalim, "Optimization of design parameters of a limited angle torque motor using analytical hierarchy process and axiomatic design theory," Production \& Manufacturing Research, vol. 2, no. 1, pp. 400-414, 2014.

[10] M. Beniakar, A. Sarigiannidis, P. Kakosimos, and A. Kladas, "Multiobjective evolutionary optimization of a surface mounted pm actuator with fractional slot winding for aerospace applications," Magnetics, IEEE Transactions on, vol. 50, no. 2, pp. 665-668, Feb 2014.

[11] F. Cupertino, G. Pellegrino, and C. Gerada, "Design of synchronous reluctance motors with multiobjective optimization algorithms," Industry Applications, IEEE
Transactions on, vol. 50, no. 6, pp. 3617-3627, Nov 2014.

[12] H. Duan and L. Gan, "Orthogonal multiobjective chemical reaction optimization approach for the brushless dc motor design," Magnetics, IEEE Transactions on, vol. 51, no. 1, pp. 1-7, 2015.

[13] R. C. Eberhart and J. Kennedy, "A new optimizer using particle swarm theory," in Proceedings of the sixth international symposium on micro machine and human science, vol. 1. New York, NY, 1995, pp. 39-43.

[14] H. Hasanien, "Particle swarm design optimization of transverse flux linear motor for weight reduction and improvement of thrust force," Industrial Electronics, IEEE Transactions on, vol. 58, no. 9, pp. 4048-4056, Sept 2011.

[15] W. Hu and G. Yen, "Adaptive multiobjective particle swarm optimization based on parallel cell coordinate system," Evolutionary Computation, IEEE Transactions on, vol. 19, no. 1, pp. 1-18, Feb 2015.

[16] S. Wu, Z. Jiao, L. Yan, Y. Shang, and C.-Y. Chen, "A new rotary voice coil motor suitable for short angular strokes-design, modeling and optimization," in Advanced Intelligent Mechatronics (AIM), 2013 IEEE/ASME International Conference on. IEEE, 2013, pp. 792-797. 